\title{
INTER-COMPANY CORRESPONDENCE
}

\section{Union Carbide Nuclear Company}

A Division of Union Carbide and Carbon Corporation

To:

$\checkmark$ A. S. Berman (5)

Copies To: Bernhardt, H. A.

Burton, D. W.

Crews, J. C.

DeMarcus, W. C. (5)

Garrett, G. A.

de la Garza, A.

Harris, W. W.

Kelly, J. P.

Levin, S. A.

Pasquier, L. P.

ORGDP Records Dept. (RC)

ORGDP Records Dept. (2)
Plant: ORGDP

Date: July 26, 1957

Subject: QUANTIZATION OF CRYSTAL VIBRATIONS

KOA-295

The Dynamics of the Crystal Lattice

The theory of the dynamics of crystal lattices was carried to a high state of perfection by Born and his co-workers around 1913. (1) In principle, these theories are vastly superior to the treatment of Debye (2) whose basic ideas will be followed in the succeeding development. However, on the practical side of the problem, the Born-von Kármán treatment is exceedingly cumbersome when numerical results are desired while the continuum model is, in some respects, quite amenable to computation. Most expositions of the Debye Theory are concerned only with the problem of counting the number of frequencies between two given frequencies for this knowledge is all that is required if one wishes only to compute the thermodynamical functions. In case it is necessary to study such phenomena as the inelastic scattering of incident gas molecules by crystals, the modes of vibration of the crystal need more specification. This memorandum contains nothing new in this respect but the original literature is scattered and some of it practically inaccessible.

(1) M. Born and Th. von Kármán: Physik. Z. 13, 297 (1912); 14, 15 (1913). (2) P. Debye: Ann. Physik 39, 789 (1912). 


\section{DISCLAIMER}

This report was prepared as an account of work sponsored by an agency of the United States Government. Neither the United States Government nor any agency Thereof, nor any of their employees, makes any warranty, express or implied, or assumes any legal liability or responsibility for the accuracy, completeness, or usefulness of any information, apparatus, product, or process disclosed, or represents that its use would not infringe privately owned rights. Reference herein to any specific commercial product, process, or service by trade name, trademark, manufacturer, or otherwise does not necessarily constitute or imply its endorsement, recommendation, or favoring by the United States Government or any agency thereof. The views and opinions of authors expressed herein do not necessarily state or reflect those of the United States Government or any agency thereof. 


\section{DISCLAIMER}

Portions of this document may be illegible in electronic image products. Images are produced from the best available original document. 
2

In a solid elastic cube of side $L$, hence volume $L^{3}$, let $\vec{u}=(u, v, w)$ be the vector displacement at some fiducial point $x, y, z$. If the Lame constarts of the solid are $\lambda$ and $\mu^{(1)}$ the strain energy per unit volume $W$ is given by

$$
W=\frac{1}{2}\left\{(\lambda+2 \mu)(\nabla \cdot \vec{u})^{2}+\mu(\nabla \times \vec{u})^{2}\right\} .
$$

The total potential energy of the elastic solid is thus

$$
V=\int_{0}^{L} d x \int_{0}^{L} d y \int_{0}^{L} d z w .
$$

If the static density of the medium is $\rho$ the total kinetic energy will be given by

$$
T=\frac{1}{2} \rho \int_{0}^{L} d x \int_{0}^{L} d y \int_{0}^{L} d z\left[\left(\frac{\partial u}{\partial t}\right)^{2}+.\left(\frac{\partial v}{\partial t}\right)^{2}+\left(\frac{\partial \dot{w}}{\partial t}\right)^{2}\right] .
$$

We shall next express the displacement components $u, v$, and was Fourier

(1) The Lame constants bear the following relations to the usual elastic moduli:

Bulk Modulus

Young 's Modulus

Poisson's Ratio

$$
\begin{aligned}
k & =\lambda+\frac{2}{3} \mu \\
E & =\frac{\mu(3 \lambda+2 \mu)}{\lambda^{\prime}+\mu} \\
& =\frac{\lambda}{2(\lambda+\mu)}
\end{aligned}
$$

Shear Modulus

218

2 
Series:

$$
\begin{aligned}
& u=\left(\frac{2}{L}\right)^{3 / 2} \sum_{f g h} a_{f g h}(t) \sin \frac{f \pi x}{L} \sin \frac{g \pi y}{L} \sin \frac{h \pi z}{L} \\
& v=\left(\frac{2}{L}\right)^{3 / 2} \sum_{f g h} b_{f g h}(t) \sin \frac{f \pi x}{L} \sin \frac{g \pi y}{L} \sin \frac{h \pi z}{L} \\
& w=\left(\frac{2}{L}\right)^{3 / 2} \sum_{f g h} c_{f g h}(t) \sin \frac{f \pi x}{L} \sin \frac{g \pi y}{L} \sin \frac{h \pi z}{L} .
\end{aligned}
$$

If we substitute these three series in the expression for the total kinetic energy $T$ we obtain

$$
T=\frac{1}{2} \rho \sum_{f g h}\left(\dot{a}_{f g h}^{2}+\dot{b}_{f g h}^{2}+\dot{c}_{f g h}^{2}\right),
$$

the dots indicating differentiation with respect to the time. Similarly, we can evaluate the potential energy obtaining

$$
\begin{aligned}
V=\frac{1}{2}(\lambda+2 \mu) \frac{\pi^{2}}{L^{2}} \sum_{f g h}\left(f a_{f g h}+g b_{f g h}+h c_{f g h}\right)^{2} \\
+\frac{1}{2} \mu \frac{\pi^{2}}{L^{2}} \sum_{f g h}\left\{\left(g c_{f g h}-h b_{f g h}\right)^{2}+\left(h a_{f g h}-f_{f g h}\right)^{2}+\left(f b_{f g h}-g a_{f g h}\right)^{2}\right\}
\end{aligned}
$$

(I) The weak point of the continuum theory emerges here. The Fourier Series employed implies that the surface is held rigid when often it is the surface vibrations which are of interest. Lennard-Jones and his co-workers attempt to bypass this difficulty by assuming that the motions of surface molecules are similar to those of interior molecules. In effect, he is studying a highly fictitious collision of a gas molecule with an interior point of the solid. 
It w1ld be convenient to replace the independent variables $a_{f g h}, b_{f g h}$, and $c_{f g h}$ by $\xi_{f g h}$, ' $f_{f g h}$, and $\zeta_{f g h}$ aefined as

$$
\begin{aligned}
& a_{f g h}=\frac{f \xi_{f g h}}{\sqrt{f^{2}+g^{2}+h^{2}}}+\frac{g \eta_{f g h}}{\sqrt{f^{2}+g^{2}}}+\frac{f h \xi_{f g h}}{\sqrt{\left(f^{2}+g^{2}\right)\left(f^{2}+g^{2}+h^{2}\right)}} \\
& b_{f g h}=\frac{g \xi_{f g h}}{\sqrt{f^{2}+g^{2}+h^{2}}}-\frac{f \eta_{f g h}}{\sqrt{f^{2}+g^{2}}}+\frac{g h \xi_{f g h}}{\sqrt{\left(f^{2}+g^{2}\right)\left(f^{2}+g^{2}+h^{2}\right)}} \\
& c_{f g h}= \\
& =\frac{h \xi_{f g h}}{\sqrt{f^{2}+g^{2}+h^{2}}} \sqrt{\frac{\left(f^{2}+g^{2}\right) \zeta_{f g h}}{\left(f^{2}+g^{2}\right)\left(f^{2}+g^{2}+h^{2}\right)}}
\end{aligned}
$$

In terms of the new variables the kinetic energy becomes

$$
\begin{aligned}
& T^{\prime}=\frac{1}{2} \rho \sum_{f g h}\left(\begin{array}{c}
2 \\
f g h
\end{array}+\eta_{f g h}^{2}+\zeta_{f g h}^{2}\right) \\
& V=\frac{\pi^{2}}{2 L^{2}} \sum_{f, g h}\left(f^{2}+g^{2}+h^{2}\right)\left[(\lambda+2 \mu) \xi_{f g h}^{2}+\mu\left(\eta_{f g h}^{2}+\zeta_{f g h}^{2}\right)\right] .
\end{aligned}
$$

The Fourler amplitudes $\xi \eta \zeta$ or $a b c$ are to be regarded as generalized coordinates. We may, therefore, calculate generalized momenta in the usual manner

$$
\emptyset_{f g h ; \xi}=\frac{\partial T}{\partial \dot{\xi}_{f g h}}=\rho \dot{\xi}_{f g h}
$$

and then form the classical Hamiltonian 


$$
\begin{aligned}
H=\frac{1}{2 \rho} & \sum_{f g h}\left(\Pi_{f g h ; \xi}^{2}+\Pi_{f g h ; \eta}^{2}+\Pi_{f g h ; \zeta}^{2}\right) \\
& +\frac{\pi^{2}}{2 L^{2}} \sum_{f g h}\left(f^{2}+g^{2}+h^{2}\right) \quad\left[(\lambda+2 \mu) \xi_{f g h}^{2}+\mu\left(\eta_{f g h}^{2}+\underset{f g h}{\left.\zeta_{f}^{2}\right)}\right] .\right.
\end{aligned}
$$

The classical equations of motion may then be obtained from Lagrange's equations or from Hamilton's and in any case become, or may be reduced to,

$$
\begin{aligned}
& \rho \ddot{\xi}_{f g h}=-\frac{\pi^{2}}{L^{2}}\left(f^{2}+g^{2}+h^{2}\right)(\lambda+2 \mu) \xi_{f g h} \\
& \rho \ddot{\eta}_{f g h}=-\frac{\pi^{2}}{L^{2}}\left(f^{2}+g^{2}+h^{2}\right) \mu \eta_{f g h} \\
& \rho \ddot{\zeta}_{f g h}=-\frac{\pi^{2}}{L^{2}}\left(f^{2}+g^{2}+h^{2}\right)_{\mu \zeta_{f g h}} .
\end{aligned}
$$

Thus, according to classical dymamics, $\xi_{f g h}$ ' $\eta_{f g h}$ ' $\zeta_{f g h}$ would vary sinuboidally in time with frequencles given by

$$
4 \pi^{2} v_{1}^{2}=\frac{m^{2}\left(f^{2}+g^{2}+h^{2}\right)}{L^{2} \rho}(\lambda+2 \mu)
$$

for $\xi_{f g h}$ and by

$$
4 \pi^{2} v_{2}^{2}=\frac{\pi^{2}\left(f^{2}+g^{2}+h^{2}\right)}{L^{2} \rho} \mu
$$

for both $\eta_{f g h}$ and $\zeta_{f g h}$. Since the Fourier terms of which the abc or $\xi \eta \zeta$ terms are the amplitudes represent standing waves of wave length $\boldsymbol{\Lambda}$ given by

$$
\frac{4 \pi^{2}}{\Lambda^{2}}=\frac{\pi^{2}}{L^{2}}\left(f^{2}+g^{2}+h^{2}\right)
$$


the velocity $\mathrm{v}$ of the waves we are describing satisfies either

$$
v_{1}^{2}=\frac{\lambda+2 \mu}{\rho}
$$

or

$$
v_{2}^{2}=\frac{\mu}{\rho}
$$

according to whether we are dealing with $\xi$, or with $n$ and $\zeta$. It can be easily shown that the waves arising from the $\xi$ amplitudes are the longitudinal waves while the $\eta$ and $\zeta$ waves are shear walves.

The number of frequencies less than a given frequency $v$ can be found as follows. The number of longitudinal waves with frequencies less than $v$ is the number of different ways one can form the sum $f^{2}+g^{2}+h^{2}$ from positive integers so that

$$
f^{2}+g^{2}+h^{2} \leqq \frac{4 \nu^{2} \rho \nu^{2}}{\lambda+2 \mu} .
$$

If this number is large, as it will be except for very low frequencies, the number of ways we can form the sum is given by one-eighth the volume of a sphere of radius $R=\frac{2 L V}{v_{1}}$ or just

$$
\frac{\pi}{6}\left(\frac{2 L}{v_{1}}\right)^{3} v^{3}
$$

Similarly, the number of shear waves of frequency less than $v$ is

$$
\text { 2. } \frac{\pi}{6}\left(\frac{2 I}{v_{2}}\right)^{3} v^{3} \text {. }
$$

The total number of frequencies less than $v$ is thus

$$
\frac{4 \pi}{3} L^{3}\left(\frac{1}{v_{1}^{3}}+\frac{2}{v_{2}}\right) v^{3}
$$

and the number of frequencies between $v$ and $v+d v$ is $g(v) d v$ with 


$$
g(v)=4 \pi L^{3}\left(\frac{1}{v_{1}}+\frac{2}{v_{2}^{3}}\right) v^{2} .
$$

On the continuum model there is nothing to prevent $v$ going to infinity but if one remembers the atomic constitution of the solid it is realized that the total number of frequencies is only three times the number of molecules in the solid. We can incorporate this fact by "cutting off" (as did Debye) the frequencies at a maximum frequency $\nu_{m}$ given by

$$
\frac{4 \pi L^{3}}{3}\left(\frac{1}{v_{1}^{3}}+\frac{2}{v_{2}^{3}}\right) v_{m}^{3}=3 N
$$

or

$$
v_{m}=\left(\frac{3 N}{4 \pi L^{3}}\right)^{1 / 3} \bar{v}
$$

where

$$
\frac{3}{v^{3}}=\frac{1}{v_{1}}+\frac{2}{v_{2}}
$$

If the molecular mass is $\mathrm{m}$ the density of the solid, $\rho$, is given by

$$
\rho=\frac{\mathrm{Nm}}{\mathrm{L}^{3}}
$$

We may also express $\bar{v}$ in terms of a fictitious velocity, $c$, defined by

$$
c^{2}=\frac{k}{\rho}
$$

and Polsson's ratio $\sigma$. Herein $k$ is the bulk modulus of the solid. It then turns out that

$$
\nu_{m}=\left(\frac{3}{4 \pi} \frac{\rho}{\dot{m}}\right)^{1 / 3} \frac{c}{f(\sigma)} .
$$


with

$$
f(\sigma)=(1+\sigma)^{1 / 2}\left[\frac{1}{3^{5 / 2}(1-\sigma)^{3 / 2}}+\left(\frac{2}{3}\right)^{5 / 2} \cdot \frac{1}{(1-2 \sigma)^{3 / 2}}\right]^{1 / 3}
$$

or introducing the compressibility $X=1 / k$ and the molecular weight $M=N m$, where $\mathrm{N}$ is Avogadro's number, .. we obtain

$$
\begin{aligned}
v_{m} & =\left(\frac{3}{4 \pi}\right)^{1 / 3} \frac{\rho^{1 / 3}}{m^{1 / 3} x^{1 / 2} \cdot \rho^{1 / 2} f(\sigma)} \\
& =\frac{1}{f(\sigma)}\left(\frac{3 N}{4 \pi}\right)^{1 / 3} \frac{1}{M^{1 / 3} x^{1 / 2} \rho^{1 / 6}} .
\end{aligned}
$$

It is of interest to compare this with a formula due to Einstein (I) which is

$$
v_{\mathrm{mE}}=\frac{4}{3} \frac{\mathrm{k}}{\mathrm{h}} \cdot \frac{13.25 \times 10^{-4}}{\mathrm{M}^{1 / 3} \mathrm{X}^{1 / 2} \rho^{1 / 6}}
$$

which agrees. with the above if $f(\sigma)=1.423$ and would be correct for $\sigma \cong .37$,

a quite reasonable result.

Quantization

Having secured the classical Hamiltonian for the solid, quantization is then carried out by the Schrodinger method. One replaces

$$
\Pi_{f g h ; \xi} \longrightarrow-i \hbar \frac{\partial}{\partial \xi_{f g h}}
$$

and similarly for the other components of momenta. One then obtains the Schrödinger equation for an asdembly of independent oscillators whose classical frequencies are just those given above. The wave function of the crystal will (1) A. Einstein: Ann. der Physik 34, 170 (1911). 
tlien be a welghted sum over terms of the form

$$
\prod_{f g h} \psi_{\ell}\left(\xi_{f g h}\right) \psi_{m}\left(\eta_{f g h}\right) \psi_{n}\left(\zeta_{f g h}\right)
$$

where the $\psi$ functions are elgenfunctions of a harmonic osclilator in the $l$ or $m$ or $n^{\text {th }}$ state of excitation.

The Thermodynamics of the Crystal

It is evident from the foregoing that one needs only to study an assembly of independent oscillators in order to arrive at the thermodynamic functions of the solid. In this section we briefly review the results one obtains from such a study.

The partition function for an oscillator of classical frequency $v$ is

$$
z_{v}=\sum_{n=0}^{\infty} e^{-\beta\left(n+\frac{1}{2}\right) h v}=\frac{e^{-\frac{\beta}{2} h v}}{1-e^{-\beta h \nu}}
$$

wherein $\beta \mathrm{kT}=1$. The partition function for a set of $3 \mathrm{~N}$ oscillators of various frequencies $v_{i}$ is therefore

$$
z=\prod_{i=1}^{3 N} \frac{e^{-\frac{\beta}{2} h \nu_{i}}}{1-e^{\beta h v_{i}}}
$$

Taking logarithms on both sides we obtain :

$$
\text { In } z=\sum_{i=1}^{3 N} \pi\left[-\frac{\beta}{2} h v_{i}-\ln \left(1-e^{-\beta h v_{i}}\right)\right] \text {. }
$$

If, as is true for most solid specimens, the energy levels are so closely spaced as to be quasi-continuous we may introduce the frequency distribution 
function $g(v)$ defined so that $g(v)+O(\Delta v)^{2}$ is the number of frequencies between $v$ and $v+\Delta v$. Then we may write for ln $\mathrm{Z}$

$$
\ln z=-\int_{0}^{\infty} g(v)\left[\frac{\beta}{2} h v+\ln \left(1-e^{-\beta h v}\right)\right] d v \text {. }
$$

In the approximation we are using

$$
\begin{array}{ll}
g(v)=\frac{9 N}{v_{m}^{3}} v^{2} & v \leqq v_{\max .} . \\
g(v)=0 & v>v_{\max } .
\end{array}
$$

The Helmholtz Free Energy $F$ is then given by

$$
F=\frac{9 N}{v_{m}^{3}} \int_{0}^{v_{m}}\left[\frac{\beta}{2} h v+\ln \left(1-e^{-\beta h v}\right)\right] v^{2} d v
$$

If one defines the Debye Characteristic Temperature $\theta_{D}$ so that

$$
\mathrm{k} \theta_{\mathrm{D}}=\mathrm{h} v_{\mathrm{m}}
$$

straightforward thermodynamic formulas lead to the following relations expressed in terms of the integral

$$
D\left(\frac{\theta_{D}}{T}\right)=3 \frac{T^{3}}{\theta_{D}^{3}} \int_{0}^{\theta_{D} / T} \frac{x^{3} d x}{e^{i x}-1}
$$

Internal Energy $\mathrm{E}=\frac{9}{8} \mathrm{R} \theta_{\mathrm{D}}+3 \mathrm{RT} \mathrm{D}\left(\frac{\theta_{\mathrm{D}}}{\mathrm{T}}\right)$

Speciffc Heat $\quad C=3 R D\left(\frac{\theta_{D}}{T}\right)+3 R T \frac{d}{d T}\left\{D\left(\frac{\theta_{D}}{T}\right)\right\}$ 


$$
\text { Entropy } S=3 R\left\{\frac{4}{3} D\left(\frac{\theta_{D}}{T}\right)-\ln \left(1-e^{-\theta_{D} / T}\right)\right\}
$$

Helmboltz Free Energy $F=\frac{9}{8} R \theta_{D}+3 R T\left\{\ln \left(1-e^{-\theta_{D} / T} \cdot\right)-\frac{1}{3} D\left(\frac{\theta_{D}}{T T}\right)\right\}$.

The Deriwation of the Mean Square Displacement

The mean square amplitude of vibration of an atom in the solid can be obtained as a consequence of two theorems, one from statistics a one due to Bloch. (1) Bloch's theorem states that the probability that the $x$ coordinate of a one-dimensional harmonic oscillator of classical frequency $v$ and mass $\mathrm{m}$ falls between $\mathrm{x}$ and $\mathrm{x}+\mathrm{dx}$, when the oscillator is a member of an assembly at absolute temperature $\mathrm{T}$, is

$$
\left(\frac{4 \pi m v}{h} \tanh \frac{h v}{2 k T}\right)^{1 / 2} e^{-\left[\frac{4 \pi^{2} m v}{h} \tanh \frac{h v}{2 k T}\right] x^{2}} d x
$$

The theorem we require of statistics is the following: If the variables $y_{y}(1=1,2, \ldots N)$ are independent and each have a normal $(2)$ (Gaussian) probability distribution with zero means and variances $\sigma_{i}^{2}$, then the variable.

$$
Y=\sum_{i=1}^{N} a_{i} y_{i}
$$

where the $a_{i}$ are constants, is normally distributed with variance

$$
\sigma^{2}=\sum_{i=1}^{N} a_{i}^{2} \sigma_{i}^{2}
$$

(1) F. Bloch: Zeits: für Physik 74, 295 (1932)'.

(2) The varlable $\mathrm{z}$ is said to be normally distributed if the probability that $z$ lies between $z$ and $z+d z$ is given by

$\sigma^{2}$ is the variance and $m$ is the mean.

$$
\frac{e^{-\frac{(z-m)^{2}}{2 \sigma^{2}}}}{\sqrt{2 \pi} \sigma}
$$


Now from (4), the components of the displacement $u$, at a fixed point in the crystal, are linear comblinations of the independent coordinates a fgh' $b_{f g h}$, and $c_{f g h}$. The coordinates $a_{f g h}$ etc. are in turn linear combinations of the $\xi_{f g h}$ ' $\eta_{f g h}$, and $\zeta_{f g h}$ which are Independent and have Gaussian Distributions with variances

$$
\frac{h}{8 \pi^{2} \rho v_{1}} \operatorname{cthh} \frac{h v_{1}}{2 k \psi^{2}}, \quad \frac{h}{8 \pi^{2} \rho v_{2}} \operatorname{ctnh} \frac{h v_{2}}{2 k T}
$$

so that $a_{f g h}$ is normally distributed with variance

$$
\frac{h \operatorname{ctnh} \frac{h v_{1}}{2 k T}}{8 \pi^{2} \rho v_{1}} \frac{f^{2}}{f^{2}+g^{2}+h^{2}}+\frac{h \operatorname{ctnh} \frac{h v_{2}}{2 k T}}{8 \pi^{2} \rho v_{2}} \frac{g^{2}+h^{2}}{f^{2}+g^{2}+h^{2}} .
$$

Note that the density $\rho$ plays the role of mass for the crystal oscillators (c.f. eq. 10). Finally, we find that $u$ is normally distributed.with variance

$$
\begin{aligned}
& \frac{8}{L^{3}} \sum_{f g h} \sin ^{2} \frac{f \pi x}{L} \sin ^{2} \frac{g \pi y}{L} \sin ^{2} \frac{h \pi z}{L} \frac{h \operatorname{ctnh} \frac{h v_{1}}{2 k T} f^{2}}{8 \pi^{2} \rho v_{1}\left(f^{2}+g^{2}+h^{2}\right)} \\
& +\frac{8}{L^{3}} \sum_{f g h} \sin ^{2} \frac{f \pi x}{L} \sin ^{2} \frac{g \pi y}{L} \sin ^{2} \frac{h \pi z}{L} \frac{h \operatorname{ctnh} \frac{h v_{2}}{2 k T}\left(g^{2}+h^{2}\right)}{8 \pi^{2} \rho v_{2}\left(f^{2}+g^{2}+h^{2}\right)} .
\end{aligned}
$$

At any point, not top near the surface of the crystal, the trigonometric factors oscillate rapidly with changing $f, g, h$ while the temperature terms are slowly varying. We may therefore replace the trigonometric factors by their average value of $1 / 8$. We als $\ddot{\phi}$ approximate the sum over $f, g, h$ by integration and use the transformation 


$$
\begin{aligned}
\mathrm{f} & =r \cos \theta \\
\mathrm{g} & =r \sin \theta \cos \phi \\
\mathrm{h} & =r \sin \theta \sin \phi \\
\operatorname{dfdgdh} & =\mathrm{r}^{2} \sin \theta \text { ded } \phi \mathrm{r} r
\end{aligned}
$$

in terms of which the variance of the distribution function for $u$ becomes

$$
\begin{aligned}
& \frac{1}{L^{3}} \int_{0}^{2 L v_{m} / v_{1}} r^{2} d r \frac{h \operatorname{ctnh} \frac{h r V_{1}}{4 L k T}}{\left(4 \pi^{2} \rho r v_{1} / L\right)} \int_{0}^{\pi / 2} \cos ^{2} \theta \sin \theta d \theta \int_{0}^{\pi / 2} d \phi \\
& +\frac{1}{L^{3}} \int_{0}^{2 L v_{m} / v_{2}} r^{2} d r \frac{h \operatorname{ctnh} \frac{h r V_{2}}{4 L k T}}{\left(4 \pi^{2} \rho r V_{2} / L\right)} \int_{0}^{\pi / 2} \sin ^{2} \theta \sin \theta d \theta \int_{0}^{\pi / 2} d \phi \\
& =\frac{2}{3}\left(\frac{1}{v_{1}^{3}}+\frac{2}{v_{2}^{3}}\right) \frac{\frac{k}{2}^{2} T^{2}}{\pi h \rho} \int_{0}^{h v_{m} / 2 k T} x \operatorname{ctnh} x d x .
\end{aligned}
$$

On introducing the Debye Temperature $\theta_{D}=h v_{m} / k$ we may bring the vartance of $u$ to the form

$$
\sigma_{u}^{2}=\frac{3 h^{2}}{4 \pi^{2} m k \theta_{D}}\left[\frac{1}{4}+\left(\frac{T}{\theta_{D}}\right)^{2} \int_{0}^{\theta_{D} / T} \frac{\xi d \xi}{e^{\xi}-1}\right] .
$$

If $f_{0}$ is the structure factor for scattering of $x$ rays from a rigid crystal, then at temperature $T$ the actpal structure factor is $(1)$

$$
f_{0} e^{-M}
$$

with

$$
M=8 \pi^{2} \sigma_{u}^{2}\left(\frac{\sin \theta}{\lambda}\right)^{2} \text {, }
$$

(I) I, Waller and H. James: Proc. Roy. Soc, A 117, 214 (1927). 
$\lambda$ beiug the wave length of the $x$ rays and $\dot{\theta}$ the angle of scattering. This is the result deduced by Debye ${ }^{(i)}$ and Waller. (a)

WCD:arm

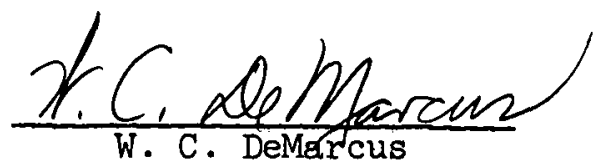

(1) P. Debye: Verh. d.. Deutsch. Phys. Ges. 15, 678, 738, 857 (1913); Annalen der Physik 43, 49 (1914). (Debye's result has an algebraic error.)

(2) I. Waller: Uppsala Dissertation (1925).

$2 \gg \quad 14$ 